This is the accepted version of the following article: De la Escosura Muñiz, A., et al. Alzheimer's disease biomarkers detection in human samples by efficient capturing through porous magnetic microspheres and labelling with electrocatalytic gold nanoparticles, in Biosensors and bioelectronics (Ed. Elsevier), vol. 67 (May 2015), p. 162-169, which has been published in final form at DOI 10.1016/j.bios.2014.07.086.

(C) 2015. This manuscript version is made available under the CC-BY-NC-ND 4.0 license http://creativecommons.org/licenses/by-nc-nd/4.0/

\title{
Alzheimer's disease biomarkers detection in human samples by efficient capturing through porous magnetic microspheres and labelling with electrocatalytic gold nanoparticles
}

Alfredo de la Escosura-Muñiz ${ }^{1}$, Zdeněk Plichta ${ }^{2}$, Daniel Horák ${ }^{2}$, Arben Merkoçi, ${ }^{1,3 *}$

${ }^{1}$ ICN2 - Nanobioelectronics \& Biosensors Group, Institut Catala de Nanociencia i

Nanotecnologia, Campus UAB, 08193 Bellaterra (Barcelona), Spain

${ }^{2}$ Institute of Macromolecular Chemistry, Academy of Sciences of the Czech Republic, Heyrovského Sq. 2, 16206 Prague 6, Czech Republic

${ }^{3}$ ICREA - Institucio Catalana de Recerca i Estudis Avançats, 08010 Barcelona, Spain

Phone number: +34937374604 ; fax number: +34935868020

E-mail: arben.merkoci@icn.cat 


\section{ABSTRACT}

A nanobiosensor based on the use of porous magnetic microspheres (PMM) as efficient capturing/pre-concentrating platform is presented for detection of Alzheimer's disease (AD) biomarkers. These PMMs prepared by a multistep swelling polymerization combined with iron oxide precipitation afford carboxyl functional groups suitable for immobilization of antibodies on the particle surface allowing an enhanced efficiency in the capturing of AD biomarkers from human serum samples. The AD biomarkers signaling is produced by gold nanoparticle (AuNP) tags monitored through their electrocatalytic effect towards hydrogen evolution reaction (HER). Novel properties of PMMs in terms of high functionality and high active area available for enhanced catalytic activity of the captured AuNPs electrocatalytic tags are exploited for the first time. A thorough characterization by scanning transmission electron microscope in high angle annular dark field mode (STEM-HAADF) demonstrates the enhanced ability of PMMs to capture a higher quantity of analyte and consequently of electrocatalytic label, when compared with commercially available microspheres. The optimized and characterized PMMs are also applied for the first time for the detection of beta amyloid and ApoE analysis at clinical relevant levels in cerebrospinal fluid (CSF), serum and plasma samples of patients suffering from AD.

\section{KEYWORDS}


Porous magnetic microspheres; Gold nanoparticles; Electrochemical immunoassay; Hydrogen evolution reaction; Alzheimer disease; Protein biomarker; ApoE; Beta-amyloid. 


\section{INTRODUCTION}

Alzheimer's disease (AD) biomarkers are neurochemical indicators used to estimate the risk or presence of this disease, which affects nowadays more than 3 million people in Europe and constitutes thus a major societal problem [Brookmeyer et al. 2007]. Although the reliable cause of most Alzheimer's cases is still essentially unknown, different studies attributed the disease evolution to the formation of abnormal deposits of beta amyloid proteins in the brain [Hardy et al. 1991]. A specific isoform of apolipoprotein, ApoE4, is a major genetic risk factor for $\mathrm{AD}$, since it also leads to an excess of amyloid formation in the brain [Polvikoski, 1995]. Due to this, both beta amyloid and ApoE4 biomarkers are considered as valid neurochemical indicators to estimate the risk or presence of the disease. Their detection in some physiological fluids such as CSF, serum and plasma can be used to diagnose AD in a very early stage and also to provide objective and reliable measures of disease progress [Marksteiner et al. 2007; Palop et al. 2010]. It has been shown that the beta amyloid biomarker shows $>80 \%$ sensitivity and specificity in distinguishing AD from dementia, which is promising for future diagnosis and treatment of AD [Bateman et al. 2006]. When used in conjunction with existing neuroimaging techniques, patients with significant memory loss developing the disease can be identified [De Meyer et al. 2010]. However, current methods and instruments of diagnostic don't fulfil the needs of early detection in such fluids with the required sensitivity and at a cost compatible with large scale screening.

In this context, nanotechnology oriented biosensors, or nanobiosensors, represent an interesting alternative for an efficient, fast, low-cost, large scaling and user-friendly diagnostics, allowing an early diagnostic and enabling thus precautionary treatment and increasing patient survival rates [Dreaden et al. 2012; De la Escosura-Muñiz and Merkoçi, 2010; Merkoci, 2010]. Of special interest are the recent approaches based on the use of 
commercially available magnetic particles for the analyte pre-concentration/purification [De la Escosura-Muñiz et al. 2010] before detection of gold nanoparticle (AuNP) tags taking advantage of their electrocatalytic activity towards Hydrogen Evolution Reaction (HER) [De la Escosura-Muñiz et al. 2009].

Magnetic microspheres used for such a purpose have to fulfill strict specifications including superparamagnetic behavior, stability in medium (no aggregation) and micrometer size range important for efficient immobilization of antibodies [Zhang et al. 2011]. To ensure uniform physical, chemical and biomedical properties, the particle size distribution should be controlled and preferably very narrow (monodisperse particles) [Shiga et al. 1996].

For this purpose, magnetic poly\{2-hydroxyethyl methacrylate-co-[2(methacryloyloxy)ethoxy]acetic acid-co-ethylene dimethacrylate ] [P(HEMA-MOEAAEDMA)] microspheres were developed by multistep swelling polymerization and precipitation of iron oxide inside pores of the particles [Horák et al. 2012, 2013]. This process was previously reported by Ugelstad for polystyrene [Ugelstad et al 1983] and later exploited by other authors for preparation of monodisperse microspheres from other monomers [Smigol et al. 1992]. Such a preparation provided robust, non-aggregated, macroporous microspheres in high yield. Their advantage consisted in absence of aromatic moieties which are contained in commercially available polystyrene particles where they induce autofluorescence disturbing cell analysis. Poly(2-hydroxyethyl methacrylate) (PHEMA) was preferred as a major component of the particles as it is frequently used in biology and medicine due to its biocompatibility, hydrophilicity and reduced non-specific protein adsorption [Wichterle et al. 1971]. Moreover, copolymerization of 2-hydroxyethyl methacrylate (HEMA) with reactive comonomers introduces carboxyl functional groups 
suitable for immobilization of biomolecules, such as proteins, antibodies and drugs, on the particle surface.

Based on these assumptions, we exploit here novel properties of porous magnetic microspheres (PMMs) when used as platforms for AD biomarkers capturing/preconcentrating from human samples and further detection in a magnetosandwich immunoassay based on the use of AuNP electrocatalytic tags. The high functionality of the novel PMMs together with their large porosity is expected to offer a high efficiency in analyte capturing and also a high active area available for enhanced catalytic activity of the captured AuNPs electrocatalytic tags. These properties are expected to improve the performance of the system in terms of sensitivity and limit of detection compared with commercially available microparticles.

\section{EXPERIMENTAL SECTION}

\subsection{Apparatus and electrodes}

A W-385 Sonicator (Heat Systems-Ultrasonics, Farmingdale, NY, USA) was used for the microsphere preparation. Immunoreactions were performed in a TS-100 Thermo shaker (BioSan, Spain). A thermostatic centrifuge (Sigma 2-16 PK, Fisher Scientific, Madrid, Spain) was used to purify the gold nanoparticle/antibody conjugates.

The electrochemical transducers were homemade screen-printed carbon electrodes (SPCEs) and the measurements were performed using an Autolab 20 (Eco-Chemie) device. See the detailed SPCE fabrication procedure in the Supporting Information.

Morphology and size of the magnetic P(HEMA-MOEAA-EDMA) microspheres, here called PMMs was determined from micrographs on a Quanta 200F scanning electron microscope (SEM; FEI; Brno, Czech Republic). The particle size distribution was characterized by the 
polydispersity index (PDI) calculated as the ratio of weight- to number-average particle diameter. The microspheres were also examined with a Paragon 1000 PC FTIR spectrometer (Perkin Elmer; Norwalk, CT, USA) with a Specac MKII Golden Gate Single Reflection ATR System with a diamond crystal and a ray angle of incidence of $45^{\circ}$.

Cross-sections of the spheres, prepared by ultramicrotomy, were examined using a Tecnai G2 F20 transmission electron microscope (TEM; FEI) in normal and in STEM-HAADF (high angle annular dark field) mode.

Elemental analysis was made on a Perkin-Elmer $2400 \mathrm{CHN}$ apparatus. The Fe content was analyzed by atomic absorption spectrometry (AAS; Perkin-Elmer 3110) of an extract from a sample obtained with dilute $\mathrm{HCl}(1: 1)$ at $80^{\circ} \mathrm{C}$ for $1 \mathrm{~h}$.

\subsection{Reagents and solutions}

2-Hydroxyethyl methacrylate (HEMA; Röhm, Darmstadt, Germany) and ethylene dimethacrylate (EDMA; Ugilor S.A., France) were vacuum distilled before use. 2(Methacryloyl)oxyethyl acetate (HEMA-Ac) was obtained from HEMA and acetic anhydride, cyclohexyl acetate (CyAc) was obtained from cyclohexanol and acetic anhydride. 2-[(Methoxycarbonyl)methoxy]ethyl methacrylate (MCMEMA) was prepared from ethylene glycol and chloracetic acid in the presence of $\mathrm{NaOH}$, producing sodium hydroxyethoxyacetate which was transformed (in the presence of $\mathrm{H}_{2} \mathrm{SO}_{4}$ and methanol) to the methylester of hydroxyethoxyacetic acid and finally to MCMEMA using methacrylic anhydride. Sodium dodecyl sulfate (SDS), benzoyl peroxide (BPO) and Methocel $90 \mathrm{HG}$ [(hydroxypropyl)methyl cellulose] were supplied by Fluka (Buchs, Switzerland).

Hydrogen tetrachloroaurate(III) trihydrate $\left(\mathrm{HAuCl}_{4} \cdot 3 \mathrm{H}_{2} \mathrm{O}, 99.9 \%\right)$, trisodium citrate, (3aminopropyl)trimethoxysilane (APS), [N-(3-dimethylamino)propyl $]-N$-ethylcarbodiimide 
(EDC), $N$-hydroxysulfosuccinimide (sulfo-NHS), phosphate buffer saline in tablet, 2-(Nmorpholino)ethanesulfonic acid (MES buffer), Tween 20, bovine serum albumin (BSA) and acetone were purchased from Sigma (Madrid, Spain).

Carboxyl- (ref. M-270) and streptavidin-modified (ref. M-280) magnetic Dynabeads ${ }^{\circledR}$ (2.8 $\mu \mathrm{m})$ were purchased from Dynal Biotech-Fisher Scientific (Madrid, Spain).

IgG from human (ref. I4506) and goat serum (ref. I5256), anti-human IgG biotin conjugate (ref. B1140, gamma chain specifically produced in goat) and anti-human IgG (ref. I1886; whole molecule produced in goat) were purchased from Sigma (Madrid, Spain).

For AD biomarkers detection, immunosandwich formats previously evaluated by ELISA tests were used. In the case of the ApoE detection, anti-ApoE capture and detection monoclonal antibodies and purified standard ApoE solution reagents contained in an ApoE ELISA kit (ref. 3712-1H-2) from Mabtech (Nacka Strand, Sweden) were used. For betaamyloid, capture monoclonal antibody (ref. A8354) from Sigma (Madrid, Spain) and detection monoclonal antibody (ref. 3740-6-1000) from Mabtech (Nacka Strand, Sweden) were selected. Standard beta amyloid solution (ref. NBP1-44967) was purchased from Novus Biologicals (Cambridge, UK).

Human cerebrospinal fluid (CSF) and plasma samples of a patient suffering from Alzheimer disease were provided by the Institute of Neurology of Ulm University (Germany).

The remaining chemicals were obtained from Sigma-Aldrich, Lachema or Penta Chemicals (Chrudim, Czech Republic) and were of analytical reagent grade. Ultrapure Q-water ultrafiltered on a Milli-Q Gradient A10 system (Millipore, Molsheim, France) was used throughout the work. 


\subsection{Methods}

Preparation of porous magnetic microspheres (PMMs)

Polystyrene (PS) seeds were obtained by emulsifier-free emulsion polymerization as described earlier [Horák et al. 2013)]. PS latex (0.3 g) was dispersed in 0.25 wt.\% SDS solution $(1 \mathrm{~mL})$ and the mixture sonicated at $15^{\circ} \mathrm{C}$ for $3 \mathrm{~min}$. The latex was mixed with an emulsion of dibutyl phthalate (DBP; $0.4 \mathrm{~g})$ in $0.25 \mathrm{wt} . \%$ SDS (1.2 ml) and 2 wt.\% $\mathrm{NaHCO}_{3}$ solutions $(0.05 \mathrm{~mL})$ under sonication at $15{ }^{\circ} \mathrm{C}$ for $4 \mathrm{~min}$. PS latex was swollen with DBP for 4 days with mild stirring $(30 \mathrm{rpm})$; swelling was repeated once more with the same amount of DBP and then four times with the double amount of DBP. The resulting PS latex contained $4.3 \mathrm{~g}$ DBP in $17.5 \mathrm{~mL}$ of the dispersion. Dispersion of DBP-swollen PS seeds $(3 \mathrm{~mL})$ in 0.25 wt.\% SDS solution was swollen in a dispersion of BPO (30 mg), HEMA-Ac (1.5 g), MCMEMA (0.3 g), EDMA (1.2 g) in $0.1 \%$ SDS solution $(7.5 \mathrm{~mL})$ for $16 \mathrm{~h}$ and transferred in a 40-mL reaction vessel. Then, dispersion of CyAc $(4 \mathrm{~g})$ in $0.1 \mathrm{wt} . \%$ SDS (10 mL) obtained with sonication $(3 \mathrm{~min})$ was added and the particles were swollen for 30 min under $\mathrm{CO}_{2}$ atmosphere. Aqueous $2 \%$ (hydroxypropyl)methyl cellulose solution $(3.5 \mathrm{~mL}$ ) was added together with 10 wt.\% Tween 20 solution $(1 \mathrm{~mL})$. Polymerization was performed at $70{ }^{\circ} \mathrm{C}$ for $16 \mathrm{~h}$ with stirring $(500 \mathrm{rpm})$ under $\mathrm{CO}_{2}$ atmosphere. The resulting macroporous P(HEMA-Ac-MCMEMA-EDMA) microspheres were filtered off and washed with 0.01 wt.\% Tween 20 and ethanol. The macroporous P(HEMA-Ac-MCMEMA-EDMA) microspheres were then hydrolyzed in $0.4 \mathrm{M} \mathrm{NaOH}(60 \mathrm{~mL})$ at $60{ }^{\circ} \mathrm{C}$ for $20 \mathrm{~h}$ under stirring (150 rpm). During hydrolysis of HEMA-Ac and MCMEMA, HEMA and [2(methacryloyloxy)ethoxy]acetic acid (MOEAA) were formed, respectively. P(HEMAMOEAA-EDMA) particles were washed with water, ethanol and water. 
In the next step, magnetic iron oxides were introduced into macroporous P(HEMA-MOEAAEDMA) microspheres. The wet microspheres (4.16 g) were imbibed by aqueous $25 \mathrm{wt} \%$ $\mathrm{FeCl}_{2}$ solution $(6 \mathrm{~mL})$ for $30 \mathrm{~min}$, the particles were separated by centrifugation, aqueous 25 wt. $\% \mathrm{FeCl}_{2}$ solution $(6 \mathrm{ml})$ again added and the particles filtered off. Separated microspheres were transferred in a $25-\mathrm{mL}$ reaction vessel, $1.35 \mathrm{M}$ ammonium solution $(5 \mathrm{~mL})$ was added, the mixture vortexed and diluted with water $(5 \mathrm{~mL})$ under Ar atmosphere. The reaction mixture was rolled on a rotator $(20 \mathrm{rpm})$ for $16 \mathrm{~h}$, the particles separated by centrifugation and washed several times with water $\left(100 \mathrm{ml}\right.$ each) to reach $\mathrm{pH}$. The formed $\mathrm{Fe}(\mathrm{OH})_{2}$ was oxidized by air oxygen for $5 \mathrm{~h}$ under stirring $(100 \mathrm{rpm})$ to form magnetite $\left(\mathrm{Fe}_{3} \mathrm{O}_{4}\right)$. The particles were again washed with water $(15 \mathrm{~mL}), 0.1 \mathrm{M} \mathrm{HCl}(6 \mathrm{~mL})$ and water. In order to increase iron oxide content in the microspheres, their imbibing with $\mathrm{FeCl}_{2}$ solution, filtration and addition of $\mathrm{NH}_{4} \mathrm{OH}$ and washing was repeated once more. During washing, peptization (formation of a colloid) occurred which removed magnetite precipitated outside of the magnetic P(HEMA-MOEAA-EDMA) microspheres (0.5 $\mathrm{g}$ of dry mass $/ \mathrm{mL}$ of wet). Microspheres were found to be stable up to 6 months when stored at $4^{\circ} \mathrm{C}$. These magnetic microspheres will be denoted in the rest of the manuscript as PMMs.

Ultramicrotomy was applied to cut the spheres into extremely thin slices that were viewed in TEM/STEM (cross-section views) for the evaluation of the content of iron oxide and/or of gold, as described in the Results and Discussion.

Preparation of gold nanoparticles (AuNPs) 
AuNPs $\left(20 \mathrm{~nm}\right.$ in diameter) were synthesized by reducing $\mathrm{HAuCl}_{4} .3 \mathrm{H}_{2} \mathrm{O}$ with trisodium citrate, following the method pioneered by Turkevich [Turkevich et al., 1951]. Briefly, all glassware used in this preparation were thoroughly cleaned in aqua regia $\left(\mathrm{HCl} / \mathrm{HNO}_{3}\right.$, volume ratio 3:1) overnight and rinsed with distilled $\mathrm{H}_{2} \mathrm{O}$ for use. Typically, a solution of 0.5 $\mathrm{mL} \mathrm{HAuCl}_{4} .3 \mathrm{H}_{2} \mathrm{O}(25 \mathrm{mM})$ in $50 \mathrm{~mL} \mathrm{Q}$-water was heated at $120{ }^{\circ} \mathrm{C}$ under stirring. Then, $1.25 \mathrm{~mL}$ of $1.1 \%$ sodium citrate was added to this solution under constant stirring. The solution was boiled during $10 \mathrm{~min}$, when the colorless solution turned violet and then changed to red. After cooling at room temperature with stirring, the solution was protected from light and stored at $4{ }^{\circ} \mathrm{C}$.

\section{AuNPs modification with antibodies}

The mechanism behind conjugation of the detection antibodies onto AuNPs surface is through adsorption that most probably occurs thanks to the presence of cysteine (which contains thiol groups) in the constant region of the antibodies that facilitates the binding (the well-known affinity of thiol groups) to gold nanoparticles. The conjugation was carried out following a previously optimized procedure [Ambrosi et al. 2007]. Briefly, $1.5 \mathrm{~mL}$ of AuNP solution in borate buffer ( $\mathrm{pH}$ 9) was incubated with $100 \mu \mathrm{L}$ of detection antibody (100 $\mu \mathrm{g} / \mathrm{mL})$ at $25{ }^{\circ} \mathrm{C}$ for $20 \mathrm{~min}$ with stirring $(600 \mathrm{rpm})$. The conjugates were then blocked by incubating the solution with $100 \mu \mathrm{L}$ of aqueous BSA solution $\left(10 \mathrm{mg} \mathrm{mL}^{-1}\right)$ and stirring was continued for other $20 \mathrm{~min}$. Finally, the solution was centrifuged at $14,000 \mathrm{rpm}$ at $4{ }^{\circ} \mathrm{C}$ for 20 min. The supernatant was removed and the AuNP pellet was redispersed in $1.5 \mathrm{~mL}$ of mQ-water.

It is important to note that the commercially available detection antibodies used for AD biomarkers detection were biotin-modified ones. Although this modification is not necessary 
for our approach, it doesn't affect the ability of antibodies to be conjugated onto AuNPs, as previously demonstrated [Ambrosi et al. 2007].

\section{Functionalization of PMM and oriented immobilization of antibodies}

Functionalization of the microspheres and antibody covalent immobilization were performed adapting a previously optimized procedure [Parolo et al. 2013]. EDC was pre-activated by mixing $133 \mu \mathrm{L}$ of EDC (10 mg mL $\mathrm{mL}^{-1}$ ) with $25 \mu \mathrm{L}$ of sulfo-NHS $\left(100 \mathrm{mg} \mathrm{mL}^{-1}\right)$ for $10 \mathrm{~min}$ (both solutions in $100 \mathrm{mM}$ MES, pH 5). After that, $842 \mu \mathrm{L}$ of $10 \mathrm{mM}$ MES (pH 5) was added forming solution 1. PMMs were 1:200 diluted with $10 \mathrm{mM}$ MES (pH 5) and $150 \mu \mathrm{L}$ of the suspension (420 $\mu \mathrm{g}$ of particles; $2.8 \mathrm{mg} \mathrm{mL}^{-1}$ ) was placed in an Eppendorf tube, two times washed in MES buffer and reconstituted in $150 \mu \mathrm{L}$ of the solution 1. The mixture was incubated at $37^{\circ} \mathrm{C}$ for $30 \mathrm{~min}$ under stirring (700 rpm).

After double washing in MES buffer, $30 \mu \mathrm{L}$ of capture antibody $\left(300 \mu \mathrm{g} \mathrm{mL}^{-1}\right)$ and $120 \mu \mathrm{L}$ of $10 \mathrm{mM}$ MES (pH 5) were added to the microspheres and the mixture incubated at $37^{\circ} \mathrm{C}$ for $1 \mathrm{~h}$ under stirring (700 rpm). After double washing in MES buffer, $150 \mu \mathrm{L}$ of $5 \%$ BSA in MES buffer was added to the microspheres and incubated first at $25^{\circ} \mathrm{C}$ for $20 \mathrm{~min}(700$ rpm) and then at $4{ }^{\circ} \mathrm{C}$ overnight.

\section{Capture of AD biomarker antigen and sandwich assay with AuNP tags}

After double washing in PBS-Tween buffer and once in PBS buffer, $150 \mu \mathrm{L}$ of AD biomarker protein standard solution (or the human fluid) was added to the microspheres and incubated at $25^{\circ} \mathrm{C}$ for 20 min under stirring $(700 \mathrm{rpm})$. After double washing in PBS-Tween buffer and once in PBS buffer, $150 \mu \mathrm{L}$ of the previously synthesized AuNPs/detection antibody 
conjugate was added to the microspheres and incubated at $25{ }^{\circ} \mathrm{C}$ for 20 min under stirring (700 rpm). The same procedure was used for the analysis of human CSF and plasma samples after diluting in PBS buffer.

The magnetic conjugate was four times washed in PBS-Tween 20 buffer, two times in PBS, once in water and reconstituted in $150 \mu \mathrm{L}$ of water.

In the determination of beta amyloid in CSF, the method of standard additions was applied for a sample 1:5 diluted with PBS buffer. Additions of 0, 20, 40, 60 and $80 \mathrm{ng} \mathrm{mL}^{-1}$ of the standard beta amyloid solution were evaluated, keeping constant the dilution of CSF.

\section{Electrochemical detection}

$25 \mu \mathrm{L}$ of the magnetic conjugate suspension was placed onto the SPCE surface (attached to a polyester sheet with a magnet on the reverse side of the working area) and $25 \mu \mathrm{L}$ of $2 \mathrm{M}$ $\mathrm{HCl}$ was added. An oxidative pretreatment at $+1.35 \mathrm{~V}$ during $60 \mathrm{~s}$ was first performed. After that, a reductive potential of $-1.0 \mathrm{~V}$ was applied during $100 \mathrm{~s}$. The hydrogen evolution catalyzed by the AuNP tags was chronoamperometrically monitored by measuring the cathodic current generated during the time. The absolute value of the current recorded at 100 $\mathrm{s}$ was chosen as the analytical signal.

\section{RESULTS AND DISCUSSION}

\subsection{Preparation and characterization of porous magnetic microspheres (PMMs)}


PMMs were obtained by multistep swelling polymerization modified according to Ugelstad [Ugelstad et al. 1983]. The microspheres contained 43 wt.\% of HEMA-Ac, 40 wt.\% of EDMA to make the particles insoluble in liquid media and to introduce macroporosity and 17 wt.\% of MCMEMA (0.5 mmol MCMEMA $\left.\mathrm{g}^{-1}\right)$ to prospectively introduce carboxyl groups. CyAc (60 wt.\% relative to organic phase) was used as a porogen. P(HEMA-AcMCMEMA-EDMA) microspheres were individual (non-aggregating), $3.9 \mu \mathrm{m}$ in diameter and with PDI = 1.01 documenting monodispersity of the particles. The high porosity of the particles was evidenced by SEM analysis (Figure 1 A-B).

\section{[Preferred position for figure 1]}

Macroporosity makes prospective penetration of iron salt solution and introduction of iron oxides inside the microspheres possible. After hydrolysis with $\mathrm{NaOH}, \mathrm{P}(\mathrm{HEMA}-\mathrm{MOEAA}-$ EDMA) microspheres were obtained. Successful completion of the hydrolysis was confirmed by the decrease in C content in P(HEMA-MOEAA-EDMA) particles compared with starting $\mathrm{P}($ HEMA-Ac-MCMEMA-EDMA) microspheres (see Table $\mathrm{S} 1$ in the Supporting Information). Another proof was provided by FTIR spectra of the particles before and after hydrolysis. Hydrolysis was confirmed by appearance of a peak at $1610 \mathrm{~cm}^{-1}$ in the spectrum of P(HEMA-MOEAA-EDMA) microspheres attributed to COONa (Figure 1C).

Porous magnetic microspheres (PMMs) were obtained by precipitation of iron oxides inside the pores of macroporous P(HEMA-MOEAA-EDMA) particles. SEM micrograph of PMMs is shown in Figure 1D. Particles remained undamaged during introduction of iron oxide inside the pores. Figure 1E shows SEM micrograph of a PMM in a more detail.

TEM micrograph of a cross-section of a PMM, prepared by ultramicrotomy, confirmed that the pores were filled with iron oxide (Figure 1F). After introduction of iron oxide, the 
particles contained $23.1 \mathrm{wt} . \% \mathrm{Fe}$ according to AAS. Such an amount is sufficient to easily and quickly attract the particles with a magnet. Size of the PMMs was slightly smaller (3.7 $\mu \mathrm{m})$ than that of initial P(HEMA-Ac-MCMEMA-EDMA) microspheres due to particle contraction after hydrolysis of HEMA-Ac and MCMEMA. Particle size distribution of PMMs did not change after the introduction of the magnetic compounds inside the pores; PDI $~ 1$ documents the monodispersity.

\subsection{PMMs as platforms for immunoassays}

PMMs were used as platforms in a sandwich immunoassay using AuNP tags and final detection taking advantage of the electrocatalytic activity of AuNPs towards the hydrogen evolution (HE) (Figure 2A). This kind of assay has been previously optimized and applied using commercial streptavidin-modified magnetic particles and biotinylated antibodies [Maltez-da Costa et al. 2010].

\section{[Preferred position for figure 2]}

In the present work, we have taken advantage of two main properties of the PMMs. On the one hand, they possess carboxyl groups which allow the covalent immobilization of antibodies through the formation of a peptide bond between these groups and the amino groups of the Lys residues of the antibodies via EDC chemistry. Furthermore, the high porosity of the PMMs is accompanied with a large surface area which is expected to increase the quantity of immobilized antibodies.

The optimum PMM concentration was first evaluated in a sandwich assay performed at 50 ng of human IgG $(\mathrm{HIgG}) \mathrm{mL}^{-1}$ chosen as model analyte. The maximum analytical signal was

obtained for $2.8 \mathrm{mg} \mathrm{mL}^{-1}$ (see Supporting Information). At this concentration, a compromise 
between the maximum amount of microspheres (and consequently of captured antigen) without blocking the electrode surface is found.

Taking into account that the $0.5 \mathrm{~g}$ of PMMs obtained in each synthesis allow to perform up to 1250 single assays (only $420 \mu \mathrm{g}$ of PMMs are necessary for each assay) and that they can be used for up to six months (as stated in methods section) we can conclude that the timeconsuming procedure for the PMMs preparation is not a practical problem which could limit their use as platforms for immunoassays.

The specificity of the assay was also evaluated for a non-specific antigen (goat $\operatorname{IgG}$ ) (see Supporting Information). Signals of the background level were obtained for all the PMMs concentrations, revealing not only the efficient blocking of the polymer microsphere surface (thanks to the BSA blocker buffer) but also the efficiency of the washing steps which allow to remove any AuNP that could enter pores of the microspheres.

The effect of the HIgG concentration on the analytical signal was evaluated (Figure 2B) finding a linear response in the range $0.5-500 \mathrm{ng} \mathrm{mL}^{-1}$ which allows to estimate a LOD of $\mathrm{HIgG}=550 \mathrm{pg} \mathrm{mL}^{-1}$ (calculated as the analyte concentration signal equal to the blank signal + three times its standard deviation; see the related calculations at the Supporting Information). The reproducibility of the method shows a relative standard deviation (RSD) of $4.1 \%\left([\mathrm{HIgG}]: 500 \mathrm{ng} \mathrm{mL}^{-1} ; \mathrm{n}=10\right)$.

The results were compared with those obtained using commercially available carboxylmodified microspheres following the same experimental procedure for the antibody immobilization through the peptide bond and further immunoassay and detection. In this case, $\mathrm{LOD}=7.1 \mathrm{ng} \mathrm{mL}^{-1}(\mathrm{RSD}: 8.3 \%)$. The better reproducibility and LOD obtained for the PMMs compared with the commercial carboxyl-modified microspheres could be attributed 
to both the higher homogeneity and porosity of the particle surface, as evidenced by the SEM images (Figure 3A-a and 3B-a).

\section{[Preferred position for figure 3]}

It would have been expected that for the same concentration of particles, smaller ones (commercial microparticles) would have yielded a higher electrochemical signal (smaller particles have higher surface areas), implying a higher number of surface atoms in contact with the working electrode surface. In addition, the quantity of PMMs used in each single assay $\left(2.8 \mathrm{mg} \mathrm{mL}^{-1}\right.$, as optimized in the study shown in figure $\mathrm{S} 3$ ) is lower than the used for the commercial microparticles $\left(3 \mathrm{mg} \mathrm{mL}^{-1}\right.$, as optimized in previous works [Ambrosi et al. 2007]). This should even improve the expected performance for the MMs. However, our findings demonstrate a better efficiency of PPMs, in contrary to the hypothesis.

The explanation to this behavior can be deduced from the studies shown in figure $3 b-d$, where STEM-HAADF analysis of a cross-section of a single microsphere after the sandwich immunoassay is performed. HAADF (high angle annular dark field) mode was selected for a better contrast. The different atomic weights of the iron and the gold allow to perfectly discriminating both materials inside the microsphere, with the AuNPs being the brighter dots in the micrograph. A high density of AuNPs is observed in the external face of the PMMs (Figure 3A-d). The AuNPs amount is considerably higher than that in the commercial microparticles (Figure 3B-d). In addition, AuNPs are also observed in the inner face of the PMMs (Figure 3A-c) while they are absent inside of the commercial particles (Figure 3B-c). This study demonstrates the ability of each single PMM to capture a higher amount of antigen and consequently of AuNP electrocatalytic tags than commercial microparticles, 
independently of their size. This last aspect is evidenced in figures $3 \mathrm{c}$ and $3 \mathrm{~d}$, where the same sized area (aprox. $600 \times 600 \mathrm{~nm}$ ) of the cut spheres is analyzed.

Energy dispersive X-ray spectroscopy (EDX) of the detailed inner areas confirms the presence of $\mathrm{Au}$ only inside of the PMMs (Figure 3A-e vs. Figure 3B-e). This also corroborates the advantageous properties induced by the microsphere porosity, which finally leads to the enhanced LOD in the HIgG electrocatalytic detection.

For comparison, commercial streptavidin-modified magnetic particles were also evaluated in the magnetosandwich immunoassay using biotin-modified antibodies for their immobilization on the particle surface (Figure $2 \mathrm{~B})$. Both the reproducibility $(\mathrm{RSD}=6.4 \%$ ) and the LOD (1.5 ng mL $\left.\mathrm{m}^{-1}\right)$ were inferior than those achieved for the PMMs, evidencing the excellent performance of the latter microspheres and the antibody immobilization through the peptide bond, with the additional advantage of no necessity of labelled capture antibodies. Overall, these results suggest that PMMs also probably offer a high active area available for enhanced catalytic activity of the captured AuNPs electrocatalytic tags.

\subsection{Sensitive electrocatalytic detection of AD biomarkers}

The optimized immunoassay with PMMs was also applied for the electrocatalytic detection of ApoE and beta amyloid AD biomarkers. Representative chronoamperograms obtained for different concentrations of beta amyloid biomarker are shown in Figure 4A.

\section{[Preferred position for figure 4]}

Calibration curves for standard solutions of both biomarkers were first performed, as shown in Figure 4B. Analytical parameters related to the linear relationship between the analytical signal and the logarithm of the biomarker concentration are summarized in Table 1. As stated 
in the Table, $\mathrm{LODs}=80 \mathrm{pg} \mathrm{mL}^{-1}$ and $19 \mathrm{pg} \mathrm{mL}^{-1}$ were obtained for ApoE and Beta amyloid, respectively, with low RSD values (estimated for a biomarker concentration of $5000 \mathrm{pg} \mathrm{\textrm {mL } ^ { - }}$ $\left.{ }^{1} ; n=10\right)$. These values are within the range of interest for AD diagnostics as discussed in the next section.

\subsection{ApoE determination in human CSF, serum and plasma samples}

The ApoE biomarker levels in human fluid samples of patients suffering from AD disease were evaluated. Since the levels of ApoE are typically in the $\mu \mathrm{g} \mathrm{mL}^{-1}$ range for CSF [Wahrle et al. 2007], serum [Vincent-Viry et al. 1998] and plasma [Taddeia et al. 1997], dilutions of the fluids with PBS buffer to a 1:5000 ratio were necessary to obtain analytical signals within the calibration curve range (Figure 4B). As matrix effects are not relevant in these highly diluted samples, the concentration of ApoE in each fluid was directly extrapolated from the calibration curve. In this way, $23 \pm 6,62 \pm 11$ and $57 \pm 9 \mu \mathrm{g} \mathrm{mL} \mathrm{m}^{-1}$ of ApoE were estimated in CSF, serum and plasma, respectively (Table 1).

\section{[Preferred position for table 1]}

These values and their correlation are in concordance with the previously reported data using standard techniques such as ELISA and western-blot analysis [Wahrle et al. 2007; VincentViry et al. 1998; Taddeia et al. 1997], microarray technology [Morales-Narváez et al. 2012] and electrochemical detection [Medina-Sánchez et al. 2014] based on commercial Quantum Dot tags.

\subsection{Beta amyloid determination in human CSF}

Analytical signals of the beta amyloid biomarker at the level of the background were obtained for both serum and plasma (Figure 4B) suggesting that the protein concentrations 
in these fluids are lower than the LOD of our method $\left(19 \mathrm{pg} \mathrm{mL}^{-1}\right)$. This is in accordance with the literature, where levels of beta amyloid in these fluids are found in the low $\mathrm{pg} \mathrm{mL}^{-1}$ level [Lee et al. 2005].

In contrast, a saturated signal was obtained for the concentrated CSF (Figure 4B). Serial dilutions in PBS buffer were performed, observing that for a 1:5 factor the analytical signal was within the detection range of our system (Figure 4C). As matrix effects are expected to play a significant role at this low dilution, the standard solution was several times added to a sample aliquot to estimate the beta amyloid levels in CSF.

Beta amyloid standards $\left(20 \mathrm{pg} \mathrm{mL}^{-1}\right)$ were added to the sample keeping constant 1:5 dilution, as detailed in Experimental Section. The gradual increase in the obtained analytical signals is proportional to the added concentration of beta amyloid according to the following linear relationship:

$$
\mathrm{i}(\mu \mathrm{A})=0.2096 \text { [added beta amyloid }]\left(\mathrm{pg} \mathrm{mL}^{-1}\right)+34 ; \mathrm{r}=0.991
$$

Extrapolating from this equation the point on the $\mathrm{x}$-axis at $\mathrm{y}=0$, the negative intercept on the $\mathrm{x}$-axis corresponds to the amount of the analyte in the analyzed sample. Considering also the RSD of the method, the estimated concentration of beta amyloid in CSF sample is $811 \pm 40$ $\mathrm{pg} \mathrm{mL} \mathrm{L}^{-1}$. This value is also in agreement with the expected one, since levels of beta amyloid in the 500-1000 $\mathrm{pg} \mathrm{mL}^{-1}$ range have been found as representative in CSF using standard methods [Gustafson et al. 2007].

\section{CONCLUSIONS}

New highly porous magnetic microspheres (PMMs) were produced by modified multistep swelling polymerization followed by precipitation of magnetic iron oxides inside the 
particles. The obtained PMMs $(\sim 3.9 \mu \mathrm{m})$ were characterized by various techniques that confirmed their narrow size distribution and homogeneous spreading of iron oxide nuclei inside the microspheres. The PMMs were shown to be well dispersed in phosphate buffer $(\mathrm{pH} 7)$ and suitable as platforms in a sandwich immunoassay.

Two are the main advantageous properties of the developed PMMs. First, they possess carboxyl groups allowing covalent bonding of antibodies through a peptide bond formation with the amino group of the Lys residues of the antibodies via EDC chemistry. Second, thanks to their high porosity the PMMs offer large surface area which in turn increases quantity of the immobilized antibodies, improving thus the performance of the system when compared with the commercial magnetic particles.

AuNP tags and electrocatalytic hydrogen evolution was used as detection signal demonstrating the solid operation and high analytical performance of the whole PMM-based biosensing system. Here the high active area offered by the PMMs also helps to enhance the catalytic activity of the captured AuNP tags. The developed device is applied in the analysis of two biomarkers: beta amyloid and ApoE in real clinical CSF, serum and plasma samples of patients suffering from Alzheimer disease.

\section{ACKNOWLEDGMENTS}

We acknowledge MINECO (Madrid) for the project MAT2011-25870 and the EU's support under FP7 contract number 246513 “NADINE”. We also acknowledge Prof. Markus Otto from Ulm University (Germany) for kindly providing AD patient's samples.

\section{REFERENCES}

Ambrosi, A., Castañeda, M.T., Killard, A.J., Smith, M.R., Alegret, S., Merkoçi, A., 2007. Anal. Chem. 79, 5232-5240. 
De la Escosura-Muñiz, A., Sánchez-Espinel, C., Díaz-Feitas, B., González-Fernández, A., Maltez-da Costa, M., Merkoçi, A. 2009. Anal. Chem. 81, 10268-10274.

De la Escosura-Muñiz, A., Merkoçi, A. 2010. Expert Opinion on Medical Diagnostics 4(1), $21-37$

De la Escosura-Muñiz, A., Maltez-da Costa, A., Sánchez Espinel, C., Díaz-Freitas, B., Fernández-Suárez, J., González-Fernández, A., Merkoçi, A. 2010. Biosens. Bioelectron. 26, 1710-1714.

De Meyer G., Shapiro F., Vanderstichele H., Vanmechelen E., Engelborghs S., De Deyn P.P., Coart E., Hansson O., Minthon L., Zetterberg H., Blennow K., Shaw L., Trojanowski J.Q. 2010. Arch. Neurol. 67, 949-956.

Dreaden, E.C., Alkilany, A.M., Huang, X., Murphy, C.J., El-Sayed, M.A. 2012. Chem. Soc. Rev. 41, 2740-2749.

Bateman, R.J., Munsell, L.Y., Morris, J.C., Swarm, R., Yarasheski, K.E., Holtzman, D.M. 2006. Nat. Med. 12, 856-861.

Brookmeyer, R., Johnson, E., Ziegler-Graham, K., Arrighi, M.H. 2007. Alzheimer's \& Dementia 3, 186-191.

Gustafson, D.R., Skoog, I., Rosengren, L., Zetterberg, H., Blennow, K. 2007. Neurol Neurosurg Psychiatry 78(5), 461-464.

Hardy, J., Allsop, D. 1991. Trends Pharmacol. Sci. 12, 383-388.

Horák, D. Kucerova, J., Korecka, L., Jankovicova, B., Palarcık, J., Mikulasek, P., Bılkova, S. 2012. Macromolecular Bioscience, 12, 647-655.

Horák, D., Svobodová, Z., Autebert, J., Coudert, B., Královec, K., Plichta, Z., Bílková, Z., Viovy, J.L. 2013. J. Biomed. Mater. Res., Part A, 101A, 23-32. 
Lee, P.H., Bang, O.Y., Hwang, E.M., Lee, J.S., Joo, U.S., Mook-Jung, I., Huh, K. 2005. J Neural Transm 112, 1371-1379.

Maltez-da Costa, M., De la Escosura-Muñiz, A., Merkoçi, A. 2010. Electrochem. Commun. $12,1501-1504$.

Merkoçi, A. 2010. Biosens. Bioelectron. 26, 1164-1177.

Marksteiner, J., Hinterhuber, H., Humpel, C. 2007. Drugs Today 43, 423-431.

Medina-Sánchez, M., Miserere, S., Morales-Narváez, E., Merkoçi, A. 2014. Biosens. Bioelectron. 54, 279-284.

Morales-Narváez, E., Montón, H., Fomicheva, A., Merkoçi, A. 2012. Anal. Chem. 2012, 84, $6821-6827$.

Palop, J.J., Mucke, L. 2010. Nat. Neurosci. 13, 812-818.

Parolo, C., De la Escosura-Muñiz, A., Polo, E., Grazú, V., de la Fuente, J.M., Merkoçi, A. 2013. ACS Appl. Mater. Interfaces 5, 10753-10759.

Polvikoski, T. 1995. N. Engl. J. Med. 333, 1242-1247.

Shiga, K., Muramatsu, N., Kondo, T. 1996. J. Pharm. Pharmacol. 48, 891-895.

Smigol, V., Svec, F. 1992. J. Appl. Polym. Sci. 46, 1439-1448.

Taddeia, K., Clarnette, R., Gandy, S.E., Martins, R.N. 1997. Neurosci. Lett. 223, 29-32.

Turkevich, J., Stevenson, P., Hillier, J., 1951. Discuss. Faraday Soc. 11, 55-75.

Ugelstad, J., Ellingsen, T., Berge, A., Helgee, B. 1983. Eur. Pat. WO 83/03920.

Vicent-Viry, M., Schiele, F., Gueguen, R., Bohnet, K., Visvikis, S. 1998. Clin. Chem. 44, 957-965.

Wichterle O. 1971. Encyclopedia of Polymer Science and Technology, Mark H.F., Gaylord N.G., Bikales N.M., Eds.; John Wiley \& Sons, New York, vol. 15.

Zhang D.H., Zhang Y.F., Zhi G.Y., Xie Y.L. 2011. Colloids Surf. B 82, 302-306. 
Wahrle, S.E, Shah, A.R., Fagan, A.M., Smemo, S., Kauwe, J.S.K., Grupe, A., Hinrichs, A., Mayo, K., Jiang, H., Thal, L.J., Goate, A.M., Holtzman, D.M. 2007. Mol. Neurodegener. 2, 7. 


\section{FIGURE AND TABLE CAPTIONS}

Fig. 1. SEM micrographs of the starting P(HEMA-Ac-MCMEMA-EDMA) microspheres (A and B) and final porous magnetic microspheres (PMMs) (D and E) and TEM micrograph of a cross-section of a PMM (F). (C) is FTIR spectrum of P(HEMA-Ac-MCMEMA-EDMA) (black line) and P(HEMA-MOEAA-EDMA) microspheres in $\mathrm{Na}^{+}$form (red line).

Fig. 2. (A) Scheme of the magnetosandwich immunoassay involving PMMs modification with antibodies, AD biomarker capture, labelling with AuNPs and electrocatalytic detection based on the hydrogen evolution reaction (HER) on screen-printed carbon electrodes (SPCEs) and a portable potentiostat. (B) Comparison of the analytical signals obtained for increasing concentrations of human $\operatorname{IgG}(\mathrm{HIgG}$, model protein) using PMMs and commercial particles modified with carboxyl groups and streptavidin.

Fig. 3. Characterization of MMs (A) and commercial particles (B) after a magnetosandwich immunoassay for $500 \mathrm{ng} \mathrm{HIgG} \mathrm{mL}^{-1}$. SEM micrographs of the particles (a) and STEMHAADF images of cross-section of a single particle (b) as well as details of the interior (c) and exterior of the particle (d). (e) Energy dispersive X-ray spectroscopy (EDX) of the interior.

Fig. 4. (A) Chronoamperograms obtained for increasing concentrations (from up to down) of beta amyloid biomarker: 0, 20, 100, 1000 and 5000 pg mL $\mathrm{mL}^{-1}$. Potential: $-1 \mathrm{~V}$, electrolyte: 1M HCl. (B) Calibration plots obtained for standard solutions of both beta amyloid and ApoE biomarkers, as well as the analytical signals obtained in human CSF, serum and plasma samples at different dilution rates. (C) Analytical signals obtained for beta amyloid in human CSF at different dilution ratios. (D) Standard additions of beta amyloid in human CSF (1:5 diluted). 
Table 1. Analytical performance of the MPM-based immunoassays in ApoE and beta amyloid detection. 


\section{FIGURE 1}
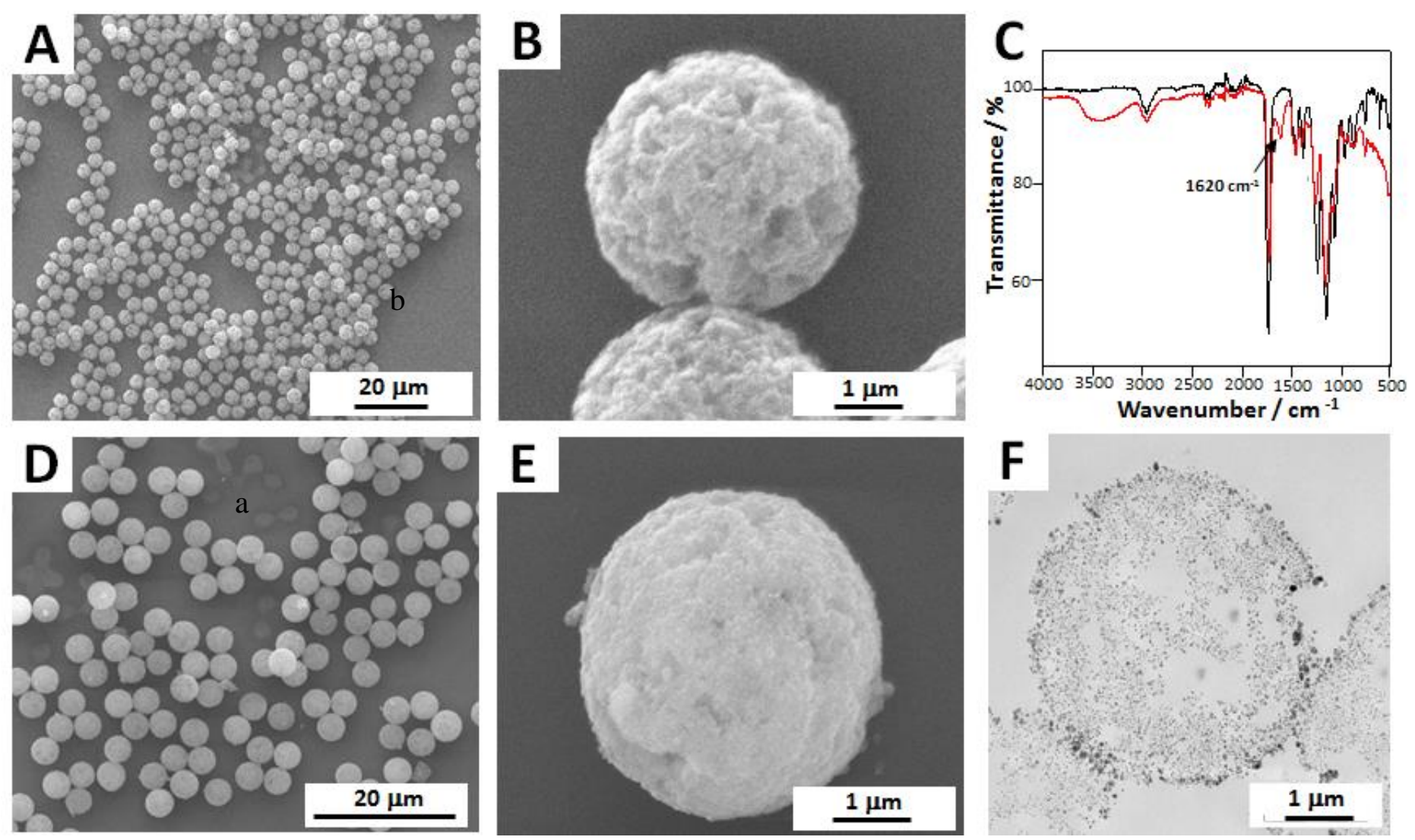


\section{FIGURE 2}
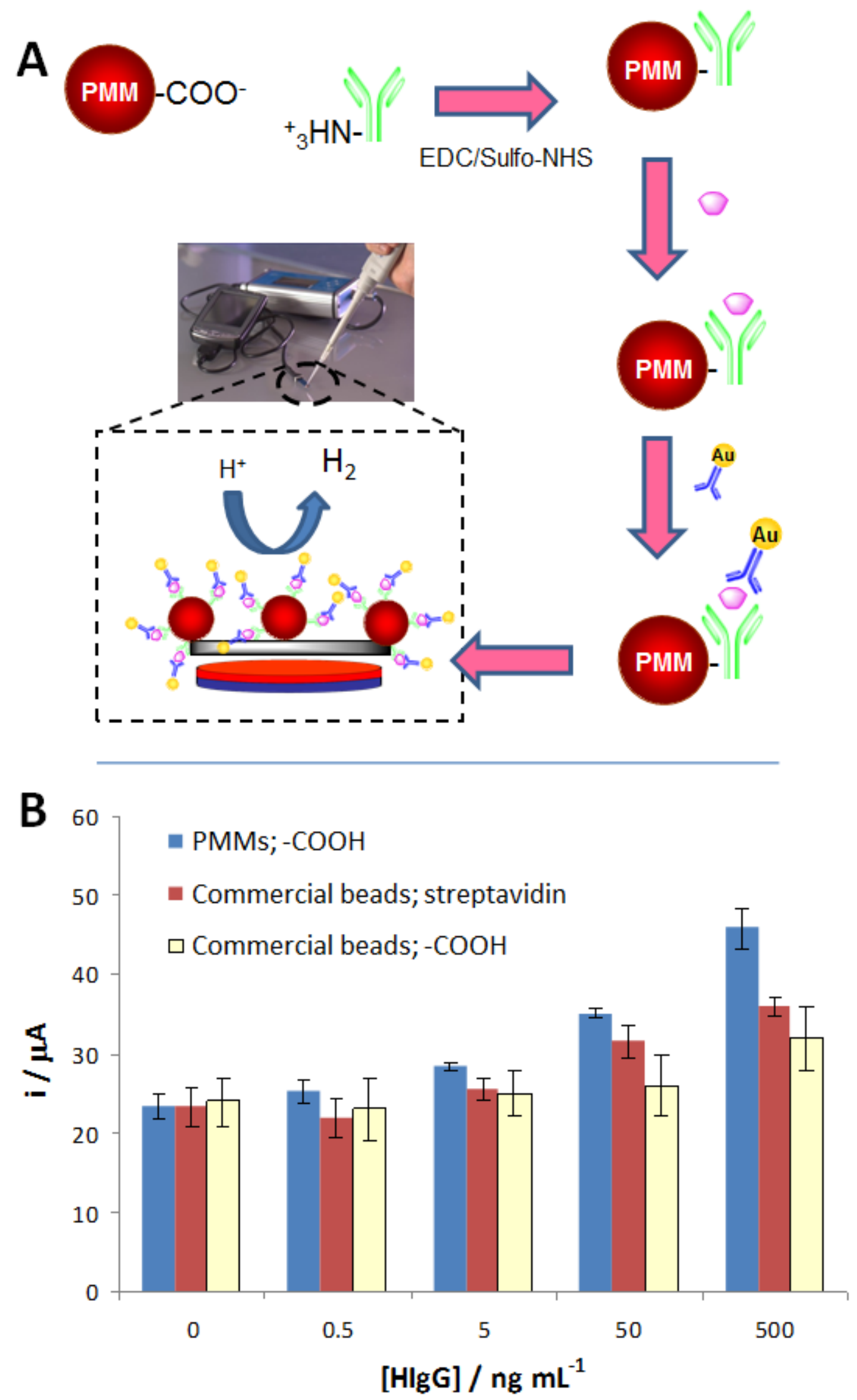
FIGURE 3
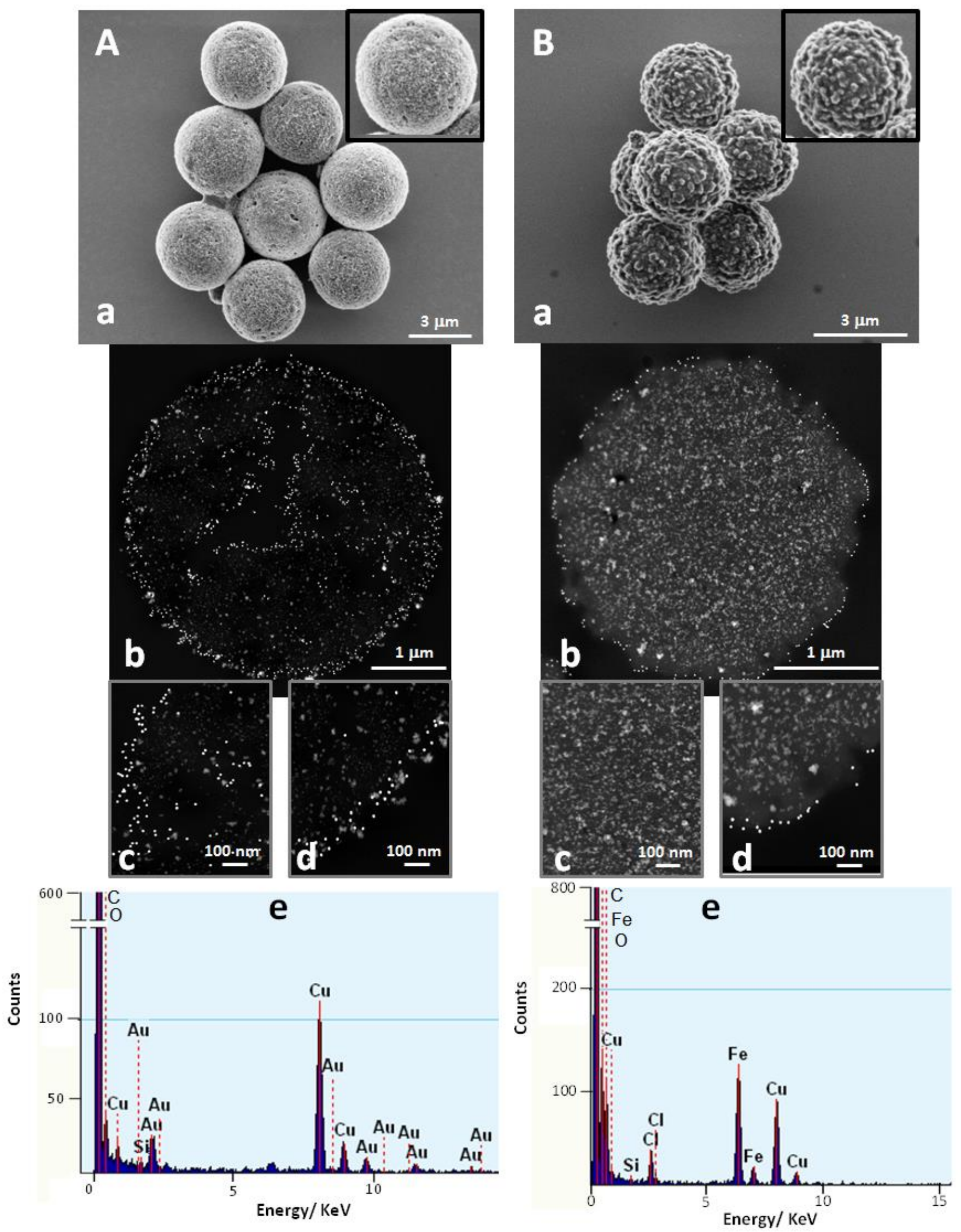


\section{FIGURE 4}
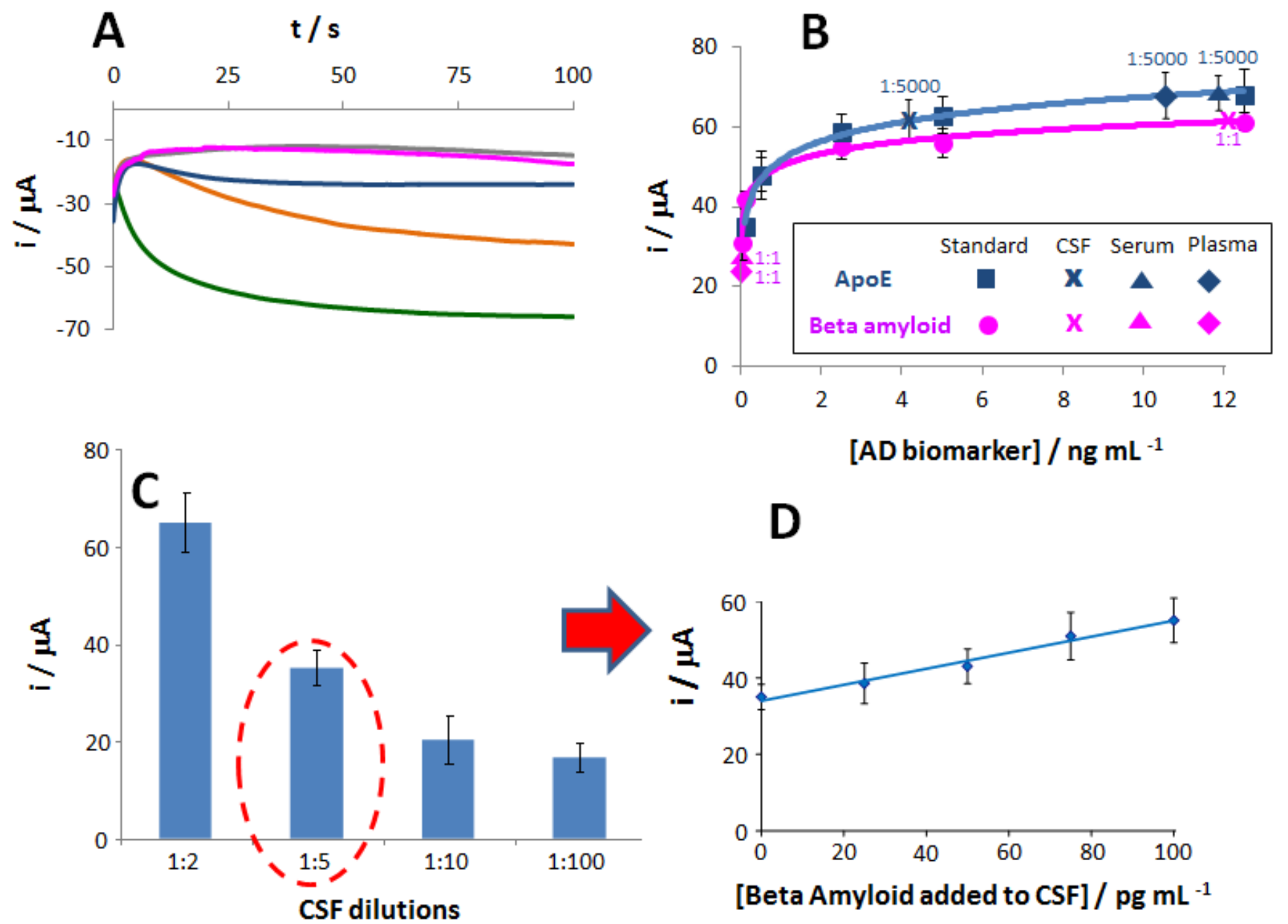


\section{TABLE 1}

\begin{tabular}{|c|c|c|}
\hline & ApoE & Beta Amyloid \\
\hline Linear range & $100-12500 \mathrm{pg} \mathrm{mL}^{-1}$ & $20-12500 \mathrm{pg} \mathrm{mL}^{-1}$ \\
\hline Equation & $\begin{array}{c}i(\mu A)=6.8347 \operatorname{Ln}[\text { ApoE }]\left(\mathrm{pg} \mathrm{mL}^{-1}\right)+4.5251 \\
r=0.997\end{array}$ & $\begin{array}{c}i(\mu \mathrm{A})=4.5768 \operatorname{Ln}\left[\text { Beta amyloid] }\left(\mathrm{pg} \mathrm{mL}^{-1}\right)+19.023\right. \\
r=0.993\end{array}$ \\
\hline RSD & $5.3 \%$ & $4.1 \%$ \\
\hline LOD & $80 \mathrm{pg} \mathrm{mL}^{-1}$ & $19 \mathrm{pg} \mathrm{mL}^{-1}$ \\
\hline CSF & $23 \pm 6 \mu \mathrm{g} \mathrm{mL}^{-1}$ & $811 \pm 40 \mathrm{pg} \mathrm{mL}^{-1}$ \\
\hline Serum & $62 \pm 11 \mu \mathrm{g} \mathrm{mL}^{-1}$ & $<19 \mathrm{pg} \mathrm{mL}^{-1}$ \\
\hline Plasma & $57 \pm 9 \mu \mathrm{g} \mathrm{mL}^{-1}$ & $<19 \mathrm{pg} \mathrm{mL}^{-1}$ \\
\hline
\end{tabular}




\section{TOC GRAPHIC}

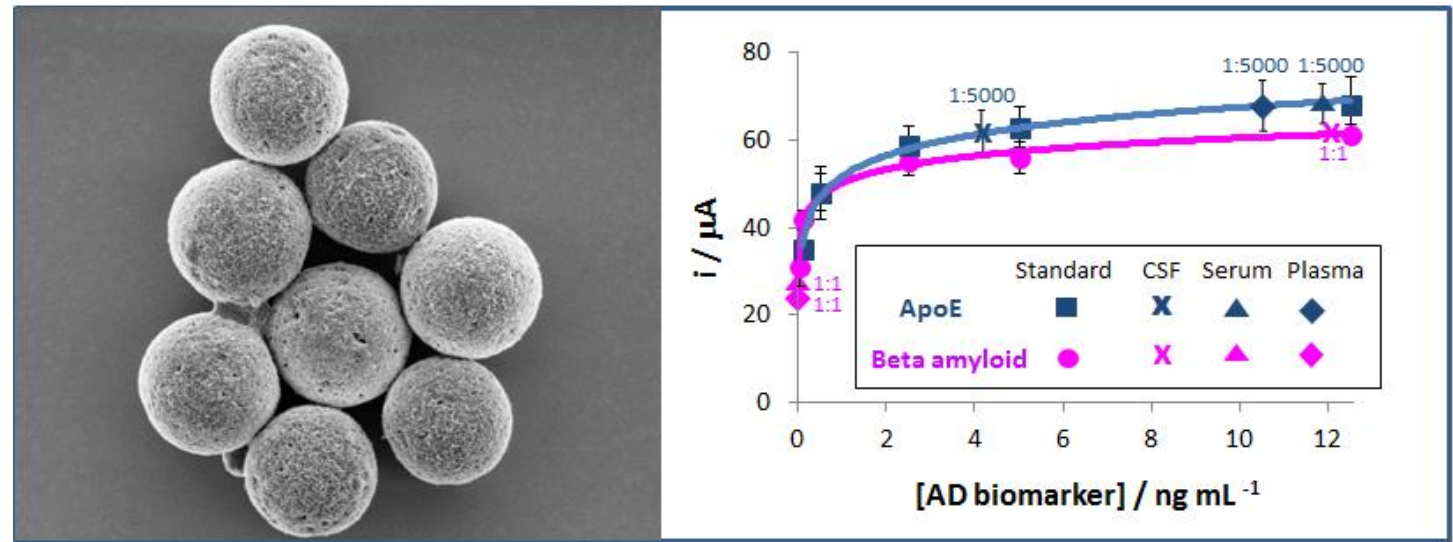

